\title{
Anwendbarkeit der BgVV-Empfehlung zum Nachweis und zur Bewertung von Listeria monocytogenes in Lebensmitteln im Rahmen der Amtlichen Lebensmittelüberwachung
}

\author{
M. Busch \\ Landesuntersuchungsanstalt für Gesundheits- und Veterinärwesen des Freistaates Sachsen
}

\section{Zusammenfassung/Erläuterung}

Mit In-Kraft-Treten der EU-VO 2073 über Mikrobiologische Kriterien in Lebensmitteln ist die weitere Anwendung o.g. BgVVEmpfehlung vom Juli 2000 in der bestehenden Fassung zu überprüfen.

1. In Anhang 1 Kapitel 1 Punkt 1.1. bis 1.3. sind sowohl die Anzahl der Proben, die zu untersuchen sind als auch die Probenmenge definiert. Diese stimmen nicht mehr mit denen in der BgVV-Empfehlung überein.

2. Für in Verkehr gebrachte Erzeugnisse während der Haltbarkeitsdauer ist außer bei Säuglingsnahrung und Lebensmittel für besondere medizinische Zwecke nur noch der quantitative Nachweis verlangt.

3. Der Grenzwert liegt bei $100 \mathrm{KbE} / \mathrm{g}$. Bisher war unter Berücksichtigung der Messunsicherheit der Grenzwert von 300 $\mathrm{KbE} / \mathrm{g}$ empfohlen worden.

Mit der vorliegenden Verordnung werden die mikrobiologischen Kriterien für bestimmte Mikroorganismen sowie die Durchführungsbestimmungen festgelegt, die von den Lebensmittelunternehmern bei der Durchführung allgemeiner und spezifischer Hygienemaßnahmen gemäß Artikel 4 der Verordnung (EG) Nr. 852/2004 einzuhalten sind.

Die zuständige Behörde überprüft die Einhaltung der in der vorliegenden Verordnung festgelegten Bestimmungen und
Kriterien gemäß der Verordnung (EG) Nr. 882/2004 unbeschadet ihres Rechts, weitere Probenahmen und Untersuchungen im Rahmen von Prozesskontrollen in Fällen, in denen der Verdacht besteht, dass Lebensmittel nicht unbedenklich sind, oder im Zusammenhang mit einer Risikoanalyse durchzuführen, um andere Mikroorganismen, deren Toxine oder Metaboliten nachzuweisen und zu messen.

Die Beurteilung derartiger Untersuchungsergebnisse im Rahmen der Amtlichen Lebensmittelüberwachung und die Einleitung entsprechender Maßnahmen sowie Befundmitteilung muss neu definiert werden.

\section{Diskussionsergebnis}

Die Bewertung von Listeria monocytogenes in Lebensmitteln erfolgt auf der Rechtsgrundlage der VO (EG) 2073/2005.

Ad-hoc-Arbeitsgruppensitzung in Kassel am 18. Juli 2006:

Die Teilnehmer haben folgenden Entwurf eines Beschlussvorschlages erarbeitet und stellen diesen zur Diskussion: Die Bewertung von Listeria monocytogenes in Lebensmitteln erfolgt auf der Rechtsgrundlage der VO (EG) 2073/2005. 